\title{
Defect Structure of High-Temperature-Grown GaMnSb/GaSb
}

\author{
P. Romanowski ${ }^{a}$, J. Bak-Misiuk ${ }^{a}$, E. Dynowska ${ }^{a}$, J.Z. Domagala $^{a}$, J. Sadowski ${ }^{a, b}$, \\ T. Wojciechowski ${ }^{a}$, A. Barcz $^{a, c}$, R. JAkiela ${ }^{a}$ And W. CAliebe ${ }^{d}$ \\ ${ }^{a}$ Institute of Physics, Polish Academy of Sciences, al. Lotników 32/46, PL-02-668 Warsaw, Poland \\ ${ }^{b}$ Lund University, MAX-lab, Ole Romers vag 1, SE-22363 Lund, Sweden \\ ${ }^{c}$ Institute of Electron Technology, al. Lotników 32/46, PL-02-668 Warsaw, Poland \\ ${ }^{d}$ HASYLAB at DESY, Notkestr. 85, D-22603 Hamburg, Germany
}

\begin{abstract}
GaMnSb/GaSb(100) layers with embedded MnSb inclusions have been grown at $720 \mathrm{~K}$ using MBE technique. This paper presents the investigation of the defect structure of $\mathrm{Ga}_{1-x} \mathrm{Mn}_{x} \mathrm{Sb}$ layers with different content of manganese (up to $x=0.07$ ). X-ray diffraction method using conventional and synchrotron radiation was applied. Dimensions and shapes of inclusions were detected by scanning electron microscopy. Depth profiles of elements were measured using secondary ion mass spectroscopy technique.
\end{abstract}

PACS numbers: 61.05.cp, 68.37.Hk, 68.49.Sf, 68.55.ag, 68.55.Ln

\section{Introduction}

GaMnSb is considered as one of new materials from the diluted magnetic semiconductor group (DMS) potentially useful for spintronics [1-4]. DMSs exhibit unique, valuable magnetic properties due to exchange interaction between carrier spins and localized spins. One of other methods for creating ferromagnetic semiconductor is introducing ferromagnetic nanoinclusions into semiconductor matrix. In order to obtain materials with desired magnetic properties it is reasonable to start with inclusions with the Curie temperature exceeding the room temperature. It was shown that high-temperature growth of GaMnSb induces the formation of MnSb inclusions in GaSb matrix, whereas low-temperature growth inhibits the formation of MnSb inclusions [3].

In order to find the best technological conditions for the formation of ferromagnetic nanoinclusions embedded in semiconductor matrix, the structural characteristics of these materials is needed. Therefore, the major purpose of this work is to determine the defect structure of high-temperature molecular beam epitaxy (MBE) grown $\mathrm{Ga}_{1-x} \mathrm{Mn}_{x} \mathrm{Sb}$ layers, in dependence on different concentration of manganese (up to $x=0.07$ ).

\section{Experimental}

$\mathrm{Ga}_{1-x} \mathrm{Mn}_{x} \mathrm{Sb}$ layers were grown on the $\mathrm{GaSb}(100)$ substrate, using the MBE technology. To achieve the creation of ferromagnetic precipitates, epitaxial growth was performed at high temperature equal to $720 \mathrm{~K}$. The thickness of $\mathrm{Ga}_{1-x} \mathrm{Mn}_{x} \mathrm{Sb}$ layers was equal to $0.63 \mu \mathrm{m}$. Three types of samples, with different Mn concentration
$\left(\mathrm{Ga}_{0.99} \mathrm{Mn}_{0.01} \mathrm{Sb}, \mathrm{Ga}_{0.97} \mathrm{Mn}_{0.03} \mathrm{Sb}\right.$ and $\left.\mathrm{Ga}_{0.93} \mathrm{Mn}_{0.07} \mathrm{Sb}\right)$ were investigated.

Strain state and the lattice parameters of $\mathrm{Ga}_{1-x} \mathrm{Mn}_{x} \mathrm{Sb}$ layers were studied using high-resolution PHILIPS-MRD diffractometer in double- and triple-axis configuration $\left(\mathrm{Cu} K_{\alpha_{1}}\right.$ radiation was used). Reciprocal space maps (RCMs) for 004 symmetrical and 224 asymmetrical reflection, as well as the rocking curves and $2 \theta / \omega$ diffraction patterns were registered.

Polycrystalline phase was investigated using monochromatic synchrotron X-ray beam $(\lambda=1.54056 \AA)$ at W1 station at HASYLAB-DESY in Hamburg, applying the glancing incidence diffraction method (coplanar $2 \theta$ scan). In this mode the rotational axis of the sample ( $\omega$ axis) has been aligned exactly with the sample surface and then the sample was rotated about this axis by a very small angle (here equal to $1^{\circ}$ ). During measurement the angular position of the sample with respect to the incident X-ray beam was fixed while the detector was rotated in the wide range of $2 \theta$ angles in the plane perpendicular to the sample surface. The coplanar $2 \theta$ scans technique is very sensitive to very thin polycrystalline layers.

To examine the depth profiles of $\mathrm{Ga}, \mathrm{Sb}$ and $\mathrm{Mn}$ elements, secondary ion mass spectrometry (SIMS) measurements were performed. Also scanning electron microscopy (SEM) was used for probing the sample surface.

\section{Results and discussion}

The SEM studies reveal the presence of isolated $\mathrm{MnSb}$ inclusions of various shapes (Fig. 1). Typical lateral dimensions depend on the Mn concentration value, and 
for the studied samples the maximum size of inclusions was about 200, 300 and $600 \mathrm{~nm}$, for $\mathrm{Ga}_{1-x} \mathrm{Mn}_{x} \mathrm{Sb}$ with $x=0.01,0.03$ and 0.07 , respectively. Rocking curve diffraction measurements ( $\omega$ scans) showed that the MnSb inclusions have block structure with the misorientation angle between blocks equal to about $1^{\circ}$.
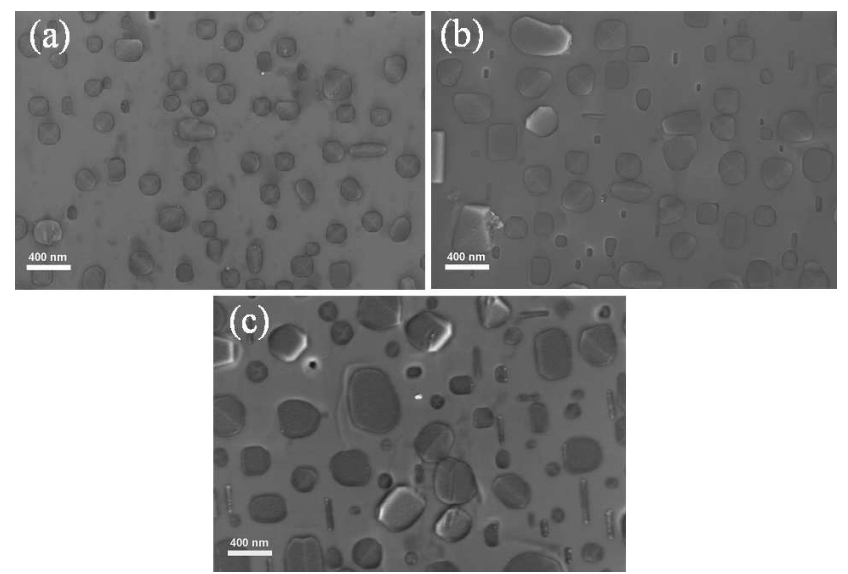

Fig. 1. SEM images of $\mathrm{Ga}_{1-x} \mathrm{Mn}_{x} \mathrm{Sb}(x=0.01$ (a), 0.03 (b), 0.07 (c)) layers grown on $\mathrm{GaSb}(100)$ substrates.

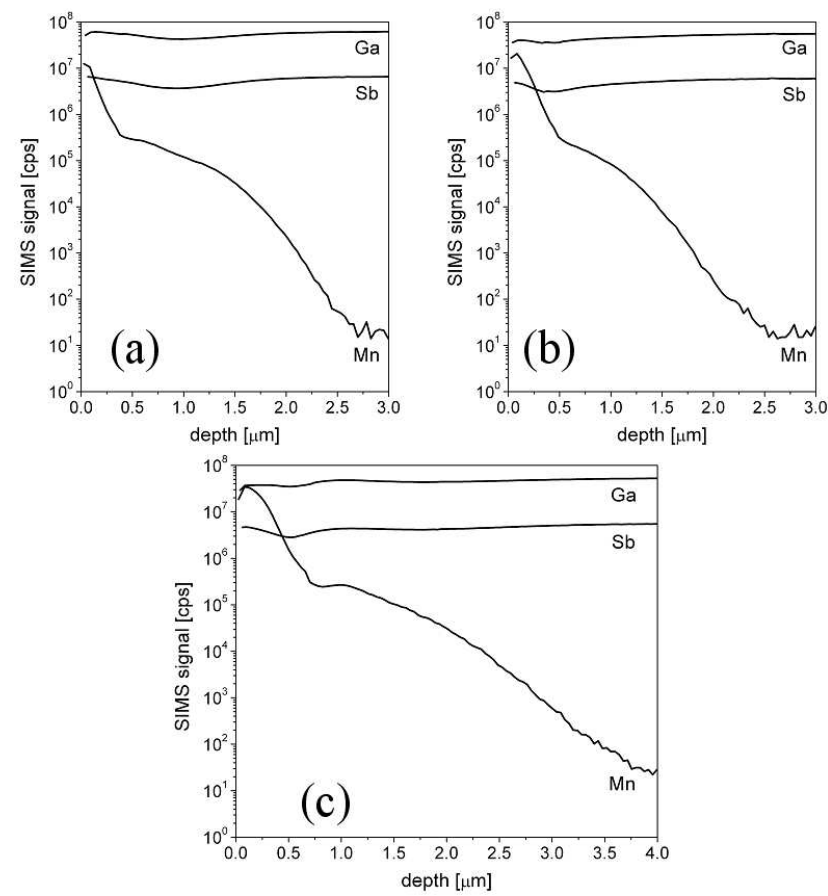

Fig. 2. SIMS depth profiles of $\mathrm{Mn}, \mathrm{Ga}$ and $\mathrm{Sb}$ in $\mathrm{Ga}_{1-x} \mathrm{Mn}_{x} \mathrm{Sb} / \mathrm{GaSb}(100)$ samples $(x=0.01$ (a), 0.03 (b), 0.07 (c)).

The SIMS studies indicate strong Mn diffusion to the GaSb substrate (Fig. 2). This process is due to the high temperature used during the MBE growth.

The polycrystalline fraction of hexagonal $\mathrm{MnSb}$ was detected by using the glancing incidence diffraction method and synchrotron radiation (Fig. 3). The different intensity may be related to the various Mn content in GaMnSb layers and also with preferred orientation of crystallites. In this measurement mode, the reflections $10.2,20.1,22.3$ and 30.0 coming from MnSb phase are visible for all three types of samples. Also for $\mathrm{Ga}_{1-x} \mathrm{Mn}_{x} \mathrm{Sb}$ with $x=0.01$ the reflections 20.0 and $21.2 \mathrm{MnSb}$ are observed as well as for $\mathrm{Ga}_{1-x} \mathrm{Mn}_{x} \mathrm{Sb}$ with $x=0.03$ the peaks coming from 10.1 and $11.1 \mathrm{MnSb}$ reflections are visible (Fig. 3).

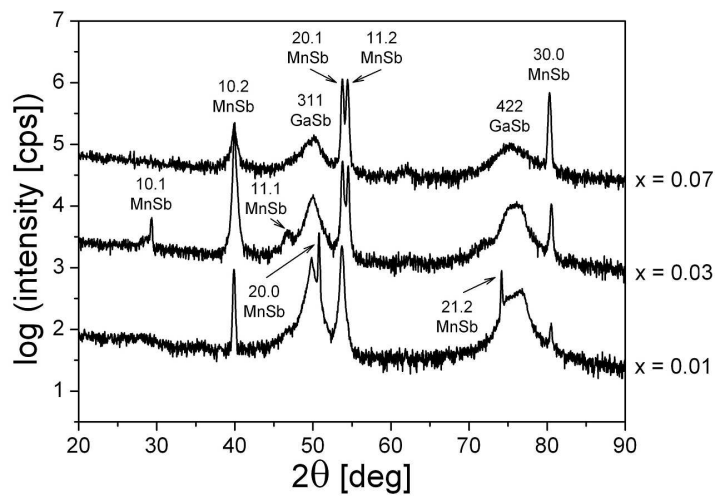

Fig. 3. Coplanar $2 \theta$ scans measured using glancing incidence diffraction geometry and X-ray synchrotron radiation source, for $\mathrm{Ga}_{1-x} \mathrm{Mn}_{x} \mathrm{Sb} / \mathrm{GaSb}(100)$ samples $(x=0.01,0.03,0.07$, respectively).

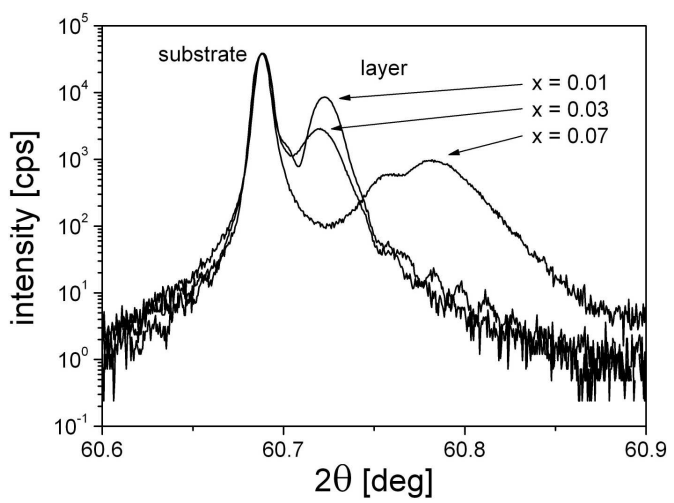

Fig. 4. $2 \theta / \omega$ diffraction patterns (004 reflection) for $\mathrm{Ga}_{1-x} \mathrm{Mn}_{x} \mathrm{Sb} / \mathrm{GaSb}(100)$ samples $(x=0.01,0.03,0.07$, respectively); high-resolution diffraction and conventional X-ray source were used.

The $2 \theta / \omega$ diffraction patterns as well as the reciprocal space maps for symmetrical reflection indicate the lattice parameter dispersion within the layers (Figs. 4 and 5). Simultaneously, pronounced diffuse scattering related to defect structure was observed for $\mathrm{Ga}_{1-x} \mathrm{Mn}_{x} \mathrm{Sb}$ with $x=0.07$. Figure 5 shows also that the lattice planes of the GaMnSb layer are parallel to the GaSb substrate planes. Reciprocal space maps for the asymmetrical reflection reveal that the investigated GaMnSb layers are 


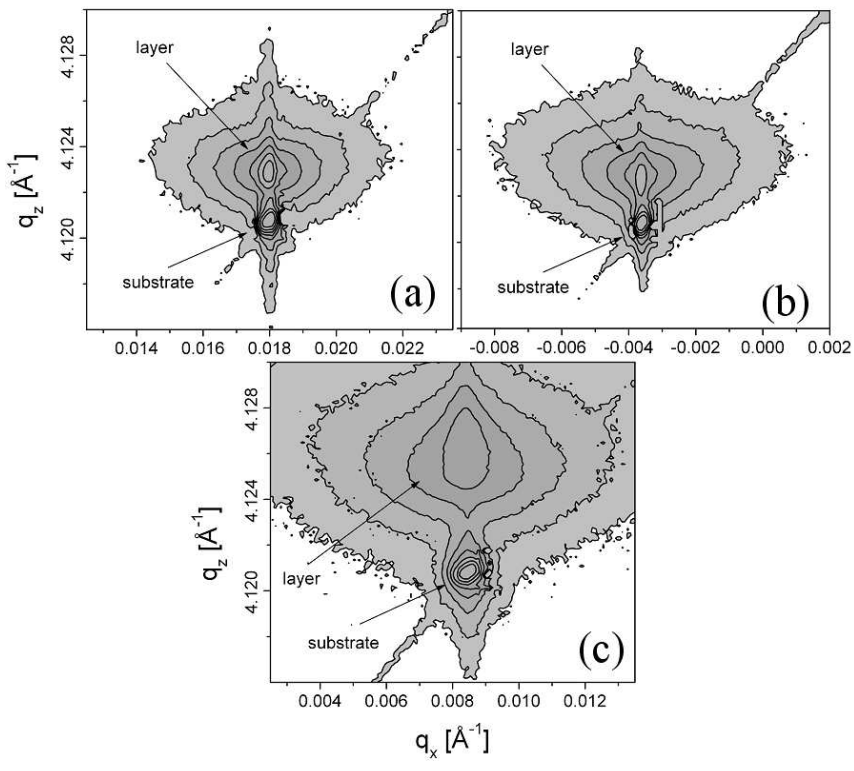

Fig. 5. Reciprocal space maps around 004 reflection for $\mathrm{Ga}_{1-x} \mathrm{Mn}_{x} \mathrm{Sb} / \mathrm{GaSb}(100)$ samples ( $x=0.01$ (a), 0.03 (b), 0.07 (c)); high-resolution diffraction and conventional X-ray source were used.

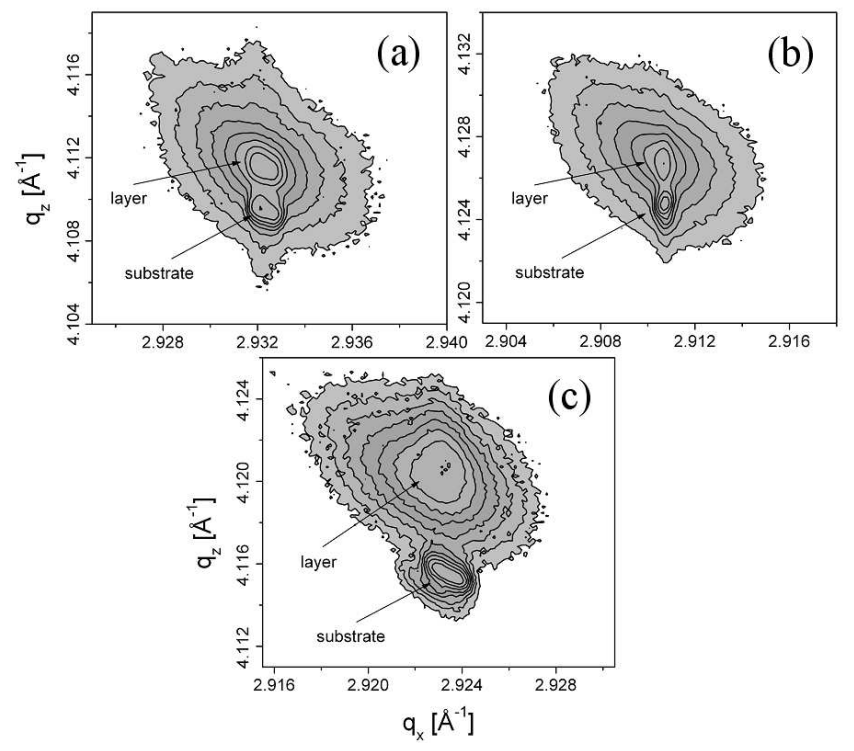

Fig. 6. Reciprocal space maps around 224 reflection for $\mathrm{Ga}_{1-x} \mathrm{Mn}_{x} \mathrm{Sb} / \mathrm{GaSb}(100)$ samples ( $x=0.01$ (a), 0.03 (b), 0.07 (c)); high-resolution diffraction and conventional X-ray source were used.

fully strained in respect of the GaSb substrate (Fig. 6) because the in-plane lattice parameter of the GaMnSb layer is equal to the GaSb lattice parameter. The out-of-plane lattice parameters for the $\mathrm{Ga}_{0.99} \mathrm{Mn}_{0.01} \mathrm{Sb}$, $\mathrm{Ga}_{0.97} \mathrm{Mn}_{0.03} \mathrm{Sb}$ and $\mathrm{Ga}_{0.93} \mathrm{Mn}_{0.07} \mathrm{Sb}$ layers are equal to $6.0922,6.0924$ and $6.0869( \pm 0.0001) \AA$, respectively. From the results we can infer that only part of $\mathrm{Mn}$ atoms form $\mathrm{MnSb}$ hexagonal inclusions, remaining fraction of $\mathrm{Mn}$ atoms, substitute the $\mathrm{Ga}$ atoms in $\mathrm{GaSb}$ layer. Due to the smaller ionic radius of Mn than that of $\mathrm{Ga}$, a decrease of the lattice parameter was detected for $\mathrm{Ga}_{1-x} \mathrm{Mn}_{x} \mathrm{Sb}$ with $x=0.07$ (Fig. 4). For $\mathrm{Ga}_{1-x} \mathrm{Mn}_{x} \mathrm{Sb}$ with $x=0.01$ and 0.03 , this effect was not observed.

\section{Summary}

The defect structure of the high-temperature MBE-grown epitaxial $\mathrm{Ga}_{1-x} \mathrm{Mn}_{x} \mathrm{Sb}$ layers on $\mathrm{GaSb}(100)$ substrate was examined. The SEM studies as well as diffraction measurements revealed the presence of hexagonal $\mathrm{MnSb}$ inclusions of different sizes related to Mn concentration. The lattice parameter values were calculated and lattice parameter dispersion was showed. The fully strained GaMnSb layers were observed. Strong Mn diffusion to the GaSb substrate was detected. For $\mathrm{Ga}_{1-x} \mathrm{Mn}_{x} \mathrm{Sb}$ with $x=0.07$ only a part of $\mathrm{Mn}$ atoms introduced to the GaSb layer forms the hexagonal $\mathrm{MnSb}$ inclusions.

\section{Acknowledgments}

This work was partially supported by national grant of Polish Ministry of Science and High Education, N202-052-32/1189 as well as by the European Union within European Regional Development Fund, through the grant Innovative Economy (POIG.01.01.02-00-008/08) and by the Foundation for Polish Science through the subsidy $12 / 2007$. The synchrotron measurements were supported by DESY/HASYLAB (EC support program: Transnational Access to Research Infrastructures) and the European Community under Contract RII3-CT-2004-506008 (IA-SFS). The MBE system at MAX-Lab, Lund used for the growth of GaMnSb is supported by the grants from the Swedish Research Council (VR).

\section{References}

[1] K. Ganesan, H.L. Bhat, J. Supercond. Nov. Magn. 21, 391 (2008).

[2] W.L. Lim, T. Wojtowicz, X. Liu, M. Dobrowolska, J.K. Furdyna, Physica E 20, 346 (2004).

[3] F. Matsukura, E. Abe, Y. Ohno, H. Ohno, Appl. Surf. Sci. 159-160, 265 (2000).

[4] C. Chen, N. Chen, L. Liu, J. Wu, Z. Liu, S. Yang, C. Chai, J. Cryst. Growth 279, 272 (2005). 